\title{
Estabilidade oxidativa em hambúrguer de frango adicionado de antioxidantes extraídos da polpa e semente da romã (Punica granatum, L.)
}

Oxidative stability in chicken hamburger with antioxidants extracted from the pulp and seed of the pomegranate (Punica granatum, L.)

\author{
J. M. Santos ${ }^{1 *}$; A. S. Borges ${ }^{2}$; P. B. L. Constant ${ }^{2}$ \\ ${ }^{1}$ Programa de Pós-Graduação em Ciência e Tecnologia de Alimentos, Universidade Federal de Sergipe, 49100-000, \\ São Cristóvão-SE, Brasil \\ ${ }^{2}$ Departamento de Tecnologia de Alimentos, Universidade Federal de Sergipe, 49100-000, São Cristóvão-SE, Brasil
}

*jideanemenezes@hotmail.com

(Recebido em 25 de junho de 2020; aceito em 01 de outubro de 2020)

\begin{abstract}
A romã (Punica granatum, L.) tornou-se uma forte alternativa no controle da oxidação lipídica em virtude de ser rica em compostos bioativos. O presente trabalho propôs avaliar a estabilidade oxidativa em hambúrguer de frango adicionado de extratos da polpa e semente de romã durante 28 dias de armazenamento a $-18{ }^{\circ} \mathrm{C}$. Foram obtidos extratos da polpa e da semente da romã, os quais tiveram os seus respectivos potenciais antioxidantes avaliados pelo método da capacidade para sequestrar o radical $\mathrm{ABTS}^{+}$ além da determinação de compostos fenólicos totais. O extrato da polpa apresentou maior teor de compostos fenólicos totais em comparação com o extrato da semente e no método $\mathrm{ABTS}^{+}$, obteve maior potencial antioxidante. $\mathrm{O}$ estudo foi realizado com quatro tratamentos: T1 (sem adição de antioxidantes), T2 (com adição de BHT), T3 (com adição de extrato da semente de romã) e T4 (com adição de extrato da polpa de romã). As dosagens para os tratamentos T2, T3 e T4 foram de $0,01 \%, 0,02 \%$ e $0,02 \%$ respectivamente. Não houve diferença significativa para os parâmetros de cor $\mathrm{L}^{*}, \mathrm{a}^{*}, \mathrm{~b}^{*}, \mathrm{C}^{*}$ e h entre os tratamentos durante o armazenamento. $\mathrm{O}$ valor de $\mathrm{pH}$ do $\mathrm{T} 1$ diferiu dos demais tratamentos a $5 \%$ de significância, os quais (T2, T3 e T4) não diferiram entre si. Na análise de TBARS o T1 apresentou diferença significativa com os demais tratamentos; T2, T3 e T4 não diferiram entre si, demonstrando que os extratos da polpa e semente de romã tiveram desempenho semelhante ao BHT no controle da oxidação lipídica.

Palavras-chave: Punica granatum, potencial antioxidante, estabilidade oxidativa
\end{abstract}

Pomegranate (Punica granatum, L.) has become a strong alternative in the control of lipid oxidation due to its rich in bioactive compounds. The present work aimed to evaluate oxidative stability in chicken burgers added from pulp extracts and pomegranate seed during 28 days of storage at $-18{ }^{\circ} \mathrm{C}$. Extracts of the pulp and seed of pomegranate were obtained, which had their respective antioxidant potentials evaluated by the method of ability to kidnap the ABTS radical in addition to the determination of total phenolic compounds. The pulp extract showed higher content of total phenolic compounds compared to seed extract and in the ABTS+ method, obtained higher antioxidant potential. The study was carried out with four treatments: T1 (without added antioxidants), T2 (with addition of BHT), T3 (with addition of pomegranate seed extract) and T4 (with addition of pomegranate pulp extract). The dosages for treatments T2, T3 and T4 were $0.01 \%$, $0.02 \%$ and $0.02 \%$ respectively. There was no significant difference for the color parameters $\mathrm{L}^{*}, \mathrm{a}^{*}, \mathrm{~b}^{*}, \mathrm{C}^{*}$ and $\mathrm{h}$ between treatments during storage. The $\mathrm{pH}$ value of $\mathrm{T} 1$ differed from the other treatments at $5 \%$ significance, which (T2, T3 and T4) did not differ from each other. In the TBARS analysis, T1 showed a significant difference with the other treatments; T2, T3 and T4 did not differ from each other, demonstrating that the extracts of the pulp and pomegranate seed had a performance similar to the BHT in the control of lipid oxidation.

Keywords: Punica granatum, antioxidant potential, oxidative stability

\section{INTRODUÇÃO}

A oxidação lipídica é um fenômeno importante que ${ }^{\circ}$ asiona a deterioração dos lipídeos e influencia na perda nutricional da carne e derivados durante a produção, processamento (cocção), 
distribuição e armazenamento, consequentemente diminuição do tempo de prateleira desses produtos, além de influenciar na aceitação do consumidor em virtude das modificações indesejáveis em suas características sensoriais decorrentes do aparecimento de aldeídos, cetonas, ácidos e alcoóis, compostos secundários oriundos da decomposição de hidroperóxidos formados nesse processo oxidativo $[1,2,3]$. Produtos cárneos oriundos da fragmentação da carne, como é o caso de hambúrgueres, são propensos a oxidação lipídica em virtude da sua elevada exposição ao oxigênio [4].

De forma geral, a carne de frango pode ser considerada uma fonte de proteína animal com baixo teor de calorias e bom valor nutricional, mesmo com os diversos fatores que influenciam na composição química, a exemplo da espécie, da alimentação, da presença ou ausência da pela e das partes do frango [5]. Apesar do baixo teor de gordura apresenta em sua composição um elevado teor de ácidos graxos poliinsaturados (PUFAs), os quais são extremamente sujeitos a reações oxidativas, em comparação com a carne de outros animais de abate $[6,7]$.

Para combater os danos ocasionados pela oxidação de lipídeos e contribuir para a estabilidade dos alimentos detentores dessas substâncias, antioxidantes sintéticos, como hidroxianisol butilado (BHA), hidroxitolueno butilado (BHT) e terbutil-hidroquinona (TBHQ) são aplicados como aditivos alimentares [8]. No entanto, pesquisas apontam para a toxidade desses aditivos alimentares o que tem levado a busca por alternativas, como a substituição por antioxidantes naturais [9]. Dessa forma, a adição de compostos bioativos com propriedades antioxidantes vem sendo largamente estudada para substituição dos antioxidantes sintéticos na indústria de alimentos. Além dos apelos em manter as propriedades sensoriais e químicas de alimentos, evitando o processo oxidativo, outras propriedades como a manutenção da saúde e prevenção de doenças estão associadas ao consumo de antioxidantes naturais [10].

Uma excelente fonte natural é a romã, planta bastante conhecida e com propriedades medicinais a qual possui, em sua composição, compostos bioativos com poder antioxidante conhecidos por proporcionar benefícios à saúde. Apresenta-se em forma de granada com diversos arilos contendo suco de cor vermelha, os quais são envolvidos em um pericarpo (casca) brilhante e semelhante a couro, coroado com um cálice persistente [11,12]. Pertence à família Punicaceae, a menor família de plantas que inclui 1 gênero e 2 espécies, incluindo as seguintes: Punica granatum (romã comestível) é originária das regiões do Irã e do Mediterrâneo e Punica protopunica (não comestível) é endógena da Socotra, ilhas no Oceano Pacífico [13, 14].

A utilização da romã e extratos obtidos a partir de partes da mesma em produtos cárneos vem sendo cada vez mais pesquisadas em virtude do seu potencial antioxidante $[4,15,16,17,18]$. Dessa forma o presente trabalho propôs avaliar a estabilidade oxidativa em hambúrguer de frango adicionado de extratos da polpa e semente de romã durante 28 dias de armazenamento a $-18{ }^{\circ} \mathrm{C}$.

\section{MATERIAL E MÉTODOS}

\subsection{Materiais}

Coxa e sobrecoxa de frango, sal, pimenta branca moída, alho em pó, cebola em pó, proteína texturizada de soja foram adquiridos em rede de supermercado, já as romãs foram obtidas no mercado local (Aracaju, Sergipe).

\subsection{Preparo dos extratos do suco e semente da romã}

As romãs foram higienizadas, cortadas em tiras com auxílio de facas e retirada manualmente os arilos (sementes contendo a polpa). Em seguida, houve a separação por meio da fricção em peneira plástica posicionada em béquer de $500 \mathrm{~mL}$. A polpa foi acondicionada em embalagem de polietileno protegida da luz e estocada em freezer à $-18{ }^{\circ} \mathrm{C}$. As sementes foram lavadas com água corrente, liofilizadas (Liotop - Liofilizador L101), trituradas manualmente com auxílio de almofariz e pistilo, embaladas com seladora a vácuo (Tec Maq - AP 500) em sacos de polietileno protegidos da luz e levadas para dessecador até o momento das extrações. 
A obtenção do extrato da polpa de romã (EPR) e extrato da semente de romã (ESR) foi realizada na proporção de 1:10; para isso foi utilizado $200 \mathrm{~mL}$ álcool etílico $97 \%$ para $20 \mathrm{~g}$ de amostra. Posteriormente a mistura foi homogeneizada com agitação magnética (Biomixer 78 HW-1) durante uma hora a temperatura ambiente, filtradas em funil de Büchner com auxílio de uma bomba de vácuo (Exipump). A fase de solvente contendo cada amostra foi drenada para balão e o álcool removido em evaporador rotativo sob vácuo (Buchi - R3), a $40^{\circ} \mathrm{C}$. O ponto final da evaporação foi a ausência de gotejamento do solvente no balão coletor. Os extratos obtidos foram acondicionados em frasco de vidro âmbar e estocados a $-18^{\circ} \mathrm{C}$.

\subsection{Compostos fenólicos totais}

A determinação do conteúdo de compostos fenólicos totais (CFT) do EPR e ESR foi realizada de acordo com o método espectrofotométrico, utilizando o reagente de Folin-Ciocalteau como agente redutor e o ácido gálico para construção da curva-padrão $(20-160 \mathrm{mg} / \mathrm{L}) .150 \mu \mathrm{L}$ de extrato foram adicionados juntamente com $300 \mu \mathrm{L}$ de solução de carbonato de sódio $\left(\mathrm{Na}_{2} \mathrm{CO}_{3}\right)$ $1 \mathrm{~N} \mathrm{e} 150 \mu \mathrm{L}$ do reagente de Folin-Ciocalteau $0,25 \mathrm{~N}$. A solução permaneceu em repouso por 60 minutos. A análise foi realizada em triplicata e a leitura realizada a $\lambda=725 \mathrm{~nm}$ (Rayleigh - UV 2601). Os resultados foram expressos em $\mathrm{mg}$ de equivalente de acido gálico/100 g de amostra (mg EAG/100g amostra) [19].

\subsection{Capacidade para sequestrar o radical 2,2'-azinobis-3-etilbenzotiazolina-6-ácido sulfônico (ABTS $\left.{ }^{+}\right)$}

A determinação da atividade antioxidante do EPR e ESR foi realizada pelo método de captura do radical $\mathrm{ABTS}^{+}$descrito no comunicado Técnico 128 [20] do Ministério da Agricultura Pecuária e Abastecimento (MAPA). As soluções estoques utilizadas foram ABTS $7 \mathrm{Mn}$ e persulfato de potássio $140 \mathrm{mM}$. O radical $\mathrm{ABTS}^{+}$foi preparado a partir da mistura das duas soluções estoque ( $88 \mu \mathrm{L}$ persulfato de potássio e 5,0 $\mathrm{mL}$ de ABTS). Posteriormente a mistura foi mantida ao abrigo da luz, em temperatura ambiente por de 16 horas. Depois de passado este período $1,0 \mathrm{~mL}$ desta mistura foi diluído em etanol até obter uma absorbância de $0,70 \mathrm{~nm} \pm 0,05$ $\mathrm{nm}$ a $734 \mathrm{~nm}$ em espectrofotômetro. $30 \mu \mathrm{L}$ dos extratos foram adicionados em tubos de ensaio contendo 3,0 mL da solução $\mathrm{ABTS}^{+}$, em seguida homogeneizado e após 6,0 min, foi realizada a leitura da absorbância em $734 \mathrm{~nm}$ usando o espectrofotômetro (Rayleigh - UV 2601). A análise foi realizada em triplicata. A curva padrão de Trolox nas concentrações de 100 e $2000 \mu \mathrm{mol} / \mathrm{L}$ foi utilizada para obtenção dos resultados. Os mesmos foram expressos em micromol equivalente de trolox/100g de amostra ( $\mu \mathrm{M} \mathrm{TE} / 100 \mathrm{~g}$ de amostra).

\subsection{Preparo dos hambúrgueres de frango}

Nessa etapa foram utilizados como matéria prima coxa e sobrecoxa de frango, proteína texturizada de soja, sal, pimenta branca moída, alho e cebola em pó [21]. A carne foi estocada a $18{ }^{\circ} \mathrm{C}$ e os demais ingredientes mantidos em local seco e arejado a temperatura ambiente.

Antes da desossa manual realizou-se o procedimento de desinfecção dos equipamentos e utensílios com solução clorada $(10 \mathrm{~mL}$ de cloro ativo a $2,5 \%$ misturado com $1 \mathrm{~L}$ de água destilada) e álcool a $70 \%$. A carne desossada foi submetida ao congelamento. Ao final de $24 \mathrm{~h}$, foi retirada do congelamento e mantida refrigerada até se obter temperatura em torno de 0 a $2^{\circ} \mathrm{C}$. Em seguida foi moída em liquidificador (Profissional - LI 2, OP). Posteriormente adicionado os demais ingredientes calculados sob o peso da massa cárnea (Tabela 1). 
Tabela 1: Ingredientes utilizados para formulação dos hambúrgueres de frango.

\begin{tabular}{lllll}
\hline & \multicolumn{4}{c}{ Formulações (\%) } \\
\cline { 2 - 5 } Ingredientes & $\mathrm{T} 1$ & $\mathrm{~T} 2$ & $\mathrm{~T} 3$ & $\mathrm{~T} 4$ \\
\hline Carne de frango & 80,00 & 80,00 & 80,00 & 80,00 \\
Pele & 10,00 & 10,00 & 10,00 & 10,00 \\
Sal & 0,71 & 0,71 & 0,71 & 0,71 \\
Pimenta branca moída & 0,14 & 0,14 & 0,14 & 0,14 \\
Alho em pó & 0,71 & 0,71 & 0,71 & 0,71 \\
Cebola em pó & 0,71 & 0,71 & 0,71 & 0,71 \\
Proteína texturizada de soja & 4,00 & 4,00 & 4,00 & 4,00 \\
Gelo & 3,73 & 3,73 & 3,73 & 3,73 \\
Antioxidante sintético BHT & - & 0,01 & - & - \\
Extrato da semente de romã (ESR) & - & - & 0,02 & - \\
Extrato da polpa de romã (EPR) & - & - & - & 0,02 \\
\hline
\end{tabular}

A massa básica para a preparação dos hambúrgueres foi dividida em quatro porções as quais receberam a adição do antioxidante de acordo com o tratamento.

Os tratamentos consistiram:

- $\quad$ T1 - hambúrgueres sem adição de antioxidante (controle negativo);

- $\quad$ T2 - hambúrgueres com adição do antioxidante sintético BHT (controle positivo);

- $\quad$ T3 - hamburgueres com adição do ESR;

- T4 - hamburgueres com adição do EPR.

A mistura foi moldada com placas de petri de $10 \mathrm{~cm}$ de diâmetro, obtendo-se hambúrgueres com peso líquido de $80 \mathrm{~g}$ cada. Os hambúrgueres foram envoltos por sacos de polietileno. Para cada tratamento foram preparados 10 hambúrgueres, os quais foram armazenados a $-18{ }^{\circ} \mathrm{C}$ por um período de até 28 dias, sendo as análises de $\mathrm{pH}$, cor instrumental e avaliação da oxidação lipídica pelo TBARS realizadas, após cocção, nos tempos 0, 7, 14, 21 e 28 dias.

\subsection{Determinação do teor de lipídeo e umidade dos hambúrgueres de frango}

As determinações de lipídeos e umidade dos hambúrgueres foram obtidas por meio da metodologia descrita pelo Instituto Adolfo Lutz (2008) [22]. Foram utilizadas nessas determinações as amostras referentes ao tempo zero de armazenamento, sendo analisadas em duplicata para o tratamento controle. Foram expressos em porcentagem (g/100g de amostra).

\subsection{Cocção dos hambúrgueres de frango}

Para as análises de $\mathrm{pH}$, cor e TBARS nos tempos $0,7,14,21$ e 28 dias, as amostras foram previamente submetidas ao processo de cocção após leve descongelamento (15 minutos em temperatura ambiente). Nos dias determinados, oito hambúrgueres, dois para cada tratamento, foram assados por 6 minutos (três minutos para cada lado) em frigideira antiaderente, a uma temperatura de $73{ }^{\circ} \mathrm{C}$ medida por meio de termômetro infravermelho (Minipa - MT 320).

\section{$2.8 \mathrm{pH}$}

Para determinação do $\mathrm{pH}$ utilizou-se o método Potenciométrico seguindo metodologia do Instituto Adolfo Lutz (2008) [22]. Após as amostras sofrerem cocção e posterior arrefecimento (30 minutos em temperatura ambiente) foi medido o $\mathrm{pH}$ das amostras (pHmetro digital microprocessado Del Lab - DLA-PH). 


\subsection{Cor instrumental}

As leituras da cor instrumental para cada tratamento foram obtidas com a utilização do colorímetro (Minolta - CR 10). No sistema CIELab, L* indica a luminosidade, a* a coordenada vermelho/verde ( $+\mathrm{a}$ indica vermelho $\mathrm{e}-\mathrm{a}$ indica verde) e $\mathrm{b}^{*}$ a coordenada amarelo/azul $(+\mathrm{b}$ indica amarelo e $-\mathrm{b}$ indica azul). Neste espaço de cor, $\mathrm{C}^{*}$ representa a saturação, e h é o ângulo de tonalidade para o sistema CIEL*C*h [23].

\subsection{Avaliação da estabilidade oxidativa pelo método TBARS}

Para quantificar as substâncias reativas ao ácido tiobarbitúrico (TBARS) foi utilizada a técnica espectrofotométrica descrita por Miyagusku et al. (2007) [24] com adaptações. Foram pesadas dez gramas de amostra em um béquer de $100 \mathrm{~mL}$. Em seguida fragmentada com auxílio de faca para facilitar a etapa de homogeneização. Colocada em um liquidificador (Profissional - LI 2, $\mathrm{OP})$ contendo $50 \mathrm{~mL}$ de água destilada e homogeneizada por 1 minuto. A mistura foi transferida para um erlenmeyer de $250 \mathrm{~mL}$ e o copo do liquidificador lavado com 47,5 ml de água destilada. $\mathrm{O}$ meio foi acidificado com $2,5 \mathrm{~mL}$ de $\mathrm{HCl} 4 \mathrm{~N}$. A mistura foi filtrada com papel filtro qualitativo Unifil - 12,5 cm de diâmetro. Uma alíquota de 5,0 $\mathrm{mL}$ foi transferida para um tubo de ensaio com tampa rosqueável em combinação com 5,0 mL de uma solução de ácido 2-tiobarbitúrico 0,02 M. Preparou-se simultaneamente o branco $(5 \mathrm{~mL}$ de água destilada $+5 \mathrm{~mL}$ do reagente TBA). Aqueceu-se os tubos de ensaio por 35 minutos em banho-maria fervente para formação da cor. A leitura da absorbância foi realizada em 532nm no espectrofotômetro (Biospectro - SP 220). O número de TBA da amostra foi expresso como miligrama $(\mathrm{mg})$ de malonaldeído por quilograma $(\mathrm{kg})$ de amostra. A curva padrão de 1,3,3-tetrametoxipropano $(0,2 \mathrm{mM})$ utilizada variou de $0,25-$ $2,5 \mathrm{~mL}$ [25]. Como o TBA reage com outras corbonilas não lipídicas presentes na amostra, como ácido ascórbico, aminoácidos e açúcares dando um falso resultado na quantificação [26]. Para minimizar possível erro da interpretação da análise com o TBA, foi realizada uma análise de amostras frescas não oxidadas e não submetidas ao congelamento, a fim de tomar conhecimento sobre as substâncias reativas com o TBA que não derivaram da oxidação de lipídeos. Dessa forma, os valores obtidos foram subtraídos dos valores das amostras assadas. Todas as análises foram realizadas em duplicata.

\subsection{Análise estatística}

Os dados das análises de compostos fenólicos totais, capacidade para sequestrar o radical $\mathrm{ABTS}^{+}, \mathrm{pH}$, cor instrumental e TBARS foram avaliados estatisticamente pelo software Statistica $^{\mathrm{TM}}$ Trial por Análise de Variância (ANOVA), teste de médias de Tukey $(\mathrm{p} \leq 0,05)$.

\section{RESULTADOS E DISCUSSÃO}

\subsection{Compostos fenólicos totais e potencial antioxidante}

Os resultados podem ser visualizados na Tabela 2. Para a determinação de CFT, os dados foram expressos em mg EAG/100g amostra. Já para a captura do radical $\mathrm{ABTS}^{+}$em $\mu \mathrm{M} \mathrm{TE} / \mathrm{g}$ amostra.

Tabela 2: Compostos Fenólicos Totais (CFT) e ABTS ${ }^{+}$para extrato da semente de romã (ESR) e extrato da polpa de romã (EPR).

\begin{tabular}{ccc}
\hline & CFT $(\mathbf{m g ~ E A G} / \mathbf{1 0 0} \mathbf{g})$ & $\mathbf{A B T S}^{+}(\boldsymbol{\mu M} \mathbf{M E} / \mathbf{1 0 0} \mathbf{g})$ \\
\cline { 2 - 3 } ESR & $241,6 \pm 18,0^{\mathrm{b}}$ & $1582,8 \pm 37,0^{\mathrm{b}}$ \\
EPR & $423,2 \pm 85,8^{\mathrm{a}}$ & $1805,3 \pm 25,3^{\mathrm{a}}$ \\
\hline
\end{tabular}

$\mathrm{n}=3$, resultados expressos em média \pm desvio-padrão. As médias seguidas pela mesma letra na coluna não diferem estatisticamente entre si $(\mathrm{p} \leq 0,05)$. 
Como mostrado na Tabela 2 o EPR apresentou maior conteúdo de fenólicos totais em comparação com o teor da ESR sendo significativo, ao nível de 5\%. Nos resultados obtidos por Vital (2014) [27] o teor de fenólicos totais no suco de romã para o extrato aquoso foi de 788,95 $\mathrm{mg} / 100 \mathrm{~g}$ em EAG e de 764,03 mg/100g em EAG no extrato hidroalcoólico. Esses resultados são superiores aos valores obtidos nesse trabalho, contudo vale ressaltar que a autora utilizou

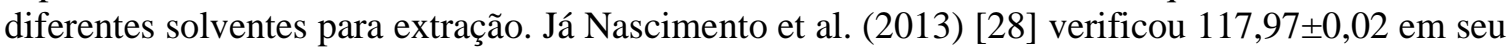
extrato etanóico de romã, um valor abaixo do observado no presente estudo.

De acordo com os resultados para o método de captura do radical $\mathrm{ABTS}^{+}$, houve diferença significativa entre o potencial antioxidante dos EPR $(1805,3 \pm 25,3 \mu \mathrm{M}$ TE/100g de amostra) e o ESR $(1582,8 \pm 37,0 \mu \mathrm{M} \mathrm{TE} / 100 \mathrm{~g}$ de amostra). Porém esses resultados são inferiores em comparação com Silva and Bezerra (2012) [29] que obteve para polpa $3500 \mu \mathrm{M}$ TE/g de amostra e para semente $600 \mu \mathrm{M}$ TE/g de amostra. Uma possível justificativa para os valores obtidos seria o emprego da agitação por um intervalo de tempo maior. Para o estudo da atividade antioxidante e conteúdo de fenólicos totais de polpas e sementes de frutas, recomenda-se agitação magnética por 4 horas ao abrigo da luz [10].

Pierotto et al. (2017) [30] em seus resultados da atividade antioxidante pelo método $\mathrm{ABTS}^{+}$ dos extratos da polpa da romã e semente de romã obteve atividade antioxidante $(48,49 \pm 4,40$ $\mu \mathrm{mol} \mathrm{ET/g} \mathrm{m.s.})$ e $(20,21 \pm 0,97 \mu \mathrm{mol} \mathrm{ET} / \mathrm{g}$ m.s. $)$ respectivamente.

\subsection{Teor de lipídeos e umidade dos hambúrgueres}

Os resultados dos teores de lipídeos e umidade das amostras de hambúrgueres de frango cruas podem ser observados na Tabela 3. Comparando com resultados encontrados na literatura, o valor da umidade $(69,63 \pm 0,31)$ encontra-se próximo aos valores obtidos por Borba et al. (2013) [31] e Torquilho et al. (2011) [32], 67,73 $\pm 0,0019$ e 65,79 $\pm 0,24$, respectivamente.

Para os resultados do teor de lipídeos das amostras de hambúrgueres, foram observados valores inferiores $(5,43 \pm 0,07)$ aos encontrados pelos mesmos autores, 11,89 $\pm 0,0044$ e 17,55 $\pm 0,55$. Uma possível justificativa seria uma maior adição de gordura na formulação desses hambúrgueres estudados anteriormente já que a legislação brasileira permite um limite máximo de $23 \%$ de adição de gordura, seja ela de origem animal ou vegetal [33].

Tabela 3: Resultados dos teores de lipídeos e umidade dos hambúrgueres (\%).

\begin{tabular}{cccc}
\hline & Media \pm DP & BORBA et al. (2013) & TORQUILHO et al. (2011) \\
\cline { 2 - 4 } Lipídeo & $5,43 \pm 0,07$ & $11,89 \pm 0,0044$ & $17,55 \pm 0,55$ \\
Umidade & $69,63 \pm 0,31$ & $67,73 \pm 0,0019$ & $65,79 \pm 0,24$ \\
\hline
\end{tabular}

$\mathrm{n}=2$, resultados expressos em média \pm desvio-padrão.

\section{$3.3 \mathrm{pH}$}

Os valores de $\mathrm{pH}$ são apresentados na Tabela 4. Observou-se que os tratamentos com adição de antioxidante, T2, T3 e T4 não apresentaram diferença significativa $(\mathrm{p}<0,05)$ entre si diferindo apenas do tratamento $\mathrm{T} 1$ (sem adição de antioxidante). Os valores das médias do $\mathrm{pH}$ para o tratamento T1 (sem antioxidante) permaneceram dentro do intervalo de 6,54 e 6,57. Já os demais tratamentos, T2 (BHT), T3 (ESR), e T4 (EPR) obtiveram médias entre 6,63 e 6,68. Verifica-se que o tratamento sem adição de antioxidante (T1), obteve valores de $\mathrm{pH}$ menores em comparação com os demais tratamentos. Em estudo semelhante com a incorporação de extrato da semente e suco de romã foi observado que os valores de $\mathrm{pH}$ de carne suína moída in natura diminuíram de 5,88 para 5,61 no período de armazenamento de 12 dias a $-4{ }^{\circ} \mathrm{C}$ [34]. 
Tabela 4: pH das amostras de hambúrgueres.

\begin{tabular}{lccccc}
\hline & \multicolumn{5}{c}{ Dias de estocagem } \\
Tratamento & $\mathbf{0}$ & $\mathbf{7}$ & $\mathbf{1 4}$ & $\mathbf{2 1}$ & $\mathbf{2 8}$ \\
\cline { 2 - 6 } T1 & $6.570 \pm 0,042^{\text {bcde }}$ & $6.555 \pm 0,007^{\mathrm{de}}$ & $6.540 \pm 0,014^{\mathrm{e}}$ & $6.565 \pm 0,007^{\mathrm{cde}}$ & $6.570 \pm 0,057^{\text {bcde }}$ \\
\hline T2 & $6.650 \pm 0,014^{\mathrm{ab}}$ & $6.630 \pm 0,014^{\mathrm{abcd}}$ & $6.680 \pm 0,028^{\mathrm{a}}$ & $6.660 \pm 0,014^{\mathrm{a}}$ & $6.665 \pm 0,021^{\mathrm{a}}$ \\
\hline T3 & $6.650 \pm 0,000^{\mathrm{ab}}$ & $6.670 \pm 0,014^{\mathrm{a}}$ & $6.665 \pm 0,007^{\mathrm{a}}$ & $6.665 \pm 0,021^{\mathrm{a}}$ & $6.665 \pm 0,007^{\mathrm{a}}$ \\
\hline T4 & $6.665 \pm 0,007^{\mathrm{a}}$ & $6.655 \pm 0,007^{\mathrm{a}}$ & $6.660 \pm 0,014^{\mathrm{a}}$ & $6.655 \pm 0,007^{\mathrm{a}}$ & $6.645 \pm 0,007^{\mathrm{abc}}$
\end{tabular}

$\mathrm{n}=2$, resultados expressos em média \pm desvio-padrão. As médias seguidas pela mesma letra não diferem estatisticamente entre si. Foi aplicado o Teste de Tukey $(\mathrm{p} \leq 0,05)$.

\subsection{Cor instrumental}

A Tabela 5 mostra os resultados para cor instrumental dos parâmetros luminosidade ( $\left.\mathrm{L}^{*}\right)$, coordenada vermelho/verde $\left(a^{*}\right)$, coordenada amarelo/azul $\left(b^{*}\right)$, saturação $\left(C^{*}\right)$ e ângulo de tonalidade (h) dos diferentes tratamentos (T1, T2, T3 e T4). De acordo com a mesma não houve diferença significativa, entre os tratamentos, dos parâmetros ao nível de 5\% de significância.

Devatkal, Narsaiah e Borah (2011) [16] em seu trabalho sobre o efeito do sal e extratos naturais, incluído da casca e semente de romã em patês de frango salgados durante o armazenamento refrigerado, obteve resultados diferentes aos encontrados. Foi verificado que o sal foi determinante na redução significativa da luminosidade $\left(\mathrm{L}^{*}\right)$ e o amarelado $\left(\mathrm{b}^{*}\right)$ das amostras, mas aumentou os valores de croma $\left(\mathrm{C}^{*}\right)$ nesse contexto os extratos provenientes da casca e semente romã não foram determinantes para modificação desses parâmetros de cor.

Tabela 5: Parâmetros para cor instrumental.

\begin{tabular}{|c|c|c|c|c|c|c|}
\hline \multirow{2}{*}{\multicolumn{2}{|c|}{ Parâmetro }} & \multicolumn{5}{|c|}{ Dias de estocagem } \\
\hline & & $\mathbf{0}$ & 7 & 14 & 21 & 28 \\
\hline \multirow[t]{4}{*}{$\mathbf{L}^{*}$} & T1 & $41,30 \pm 1,56^{\mathrm{a}}$ & $42,45 \pm 1,34^{\mathrm{a}}$ & $42,30 \pm 0,28^{a}$ & $40,95 \pm 0,35^{\mathrm{a}}$ & $42,05 \pm 0,78^{\mathrm{a}}$ \\
\hline & T2 & $41,90 \pm 0,57^{a}$ & $41,95 \pm 2,47^{\mathrm{a}}$ & $42,80 \pm 1,56^{\mathrm{a}}$ & $41,85 \pm 0,21^{\mathrm{a}}$ & $41,75 \pm 1,77^{\mathrm{a}}$ \\
\hline & T3 & $42,95 \pm 0,49^{a}$ & $42,10 \pm 1,41^{\mathrm{a}}$ & $42,25 \pm 0,92^{\mathrm{a}}$ & $41,45 \pm 1,91^{\mathrm{a}}$ & $42,05 \pm 1,63^{\mathrm{a}}$ \\
\hline & T4 & $41,60 \pm 2,12^{\mathrm{a}}$ & $41,50 \pm 0,71^{\mathrm{a}}$ & $42,90 \pm 0,28^{\mathrm{a}}$ & $42,60 \pm 1,27^{\mathrm{a}}$ & $41,05 \pm 1,34^{\mathrm{a}}$ \\
\hline \multirow[t]{4}{*}{$a^{*}$} & T1 & $+10,25 \pm 0,92^{\mathrm{a}}$ & $+10,30 \pm 0,71^{\mathrm{a}}$ & $+10,30 \pm 1,41^{\mathrm{a}}$ & $+10,05 \pm 1,63^{\mathrm{a}}$ & $+9,9 \pm 1,41^{\mathrm{a}}$ \\
\hline & T2 & $+9,70 \pm 0,42^{\mathrm{a}}$ & $+9,75 \pm 1,77^{\mathrm{a}}$ & $+9,80 \pm 0,14^{\mathrm{a}}$ & $+10,35 \pm 0,78^{\mathrm{a}}$ & $+10,35 \pm 0,92^{\mathrm{a}}$ \\
\hline & T3 & $+9,95 \pm 1,34^{\mathrm{a}}$ & $+10,40 \pm 0,85^{\mathrm{a}}$ & $+10,55 \pm 0,49^{\mathrm{a}}$ & $+9,65 \pm 0,21^{\mathrm{a}}$ & $+10,30 \pm 0,99^{\mathrm{a}}$ \\
\hline & T4 & $+9,50 \pm 1,41^{\mathrm{a}}$ & $+10,25 \pm 1,20^{\mathrm{a}}$ & $+10,05 \pm 0,35^{\mathrm{a}}$ & $+9,80 \pm 0,28^{\mathrm{a}}$ & $+9,65 \pm 0,78^{\mathrm{a}}$ \\
\hline \multirow{4}{*}{$\mathbf{b}^{*}$} & T1 & $+30,45 \pm 0,78^{a}$ & $+29,70 \pm 3,25^{\mathrm{a}}$ & $+30,00 \pm 1,56^{a}$ & $+30,80 \pm 0,99^{\mathrm{a}}$ & $+29,55 \pm 1,06^{a}$ \\
\hline & T2 & $+30,15 \pm 1,20^{\mathrm{a}}$ & $+29,50 \pm 0,71^{\mathrm{a}}$ & $+30,50 \pm 0,99^{\mathrm{a}}$ & $+30,20 \pm 0,71^{\mathrm{a}}$ & $+29,75 \pm 0,64^{\mathrm{a}}$ \\
\hline & T3 & $+30,05 \pm 1,48^{\mathrm{a}}$ & $+30,00 \pm 0,71^{\mathrm{a}}$ & $+30,05 \pm 0,92^{\mathrm{a}}$ & $+30,40 \pm 0,85^{\mathrm{a}}$ & $+30,35 \pm 1,20^{\mathrm{a}}$ \\
\hline & T4 & $+30,45 \pm 0,78^{a}$ & $+30,15 \pm 0,21^{\mathrm{a}}$ & $+30,40 \pm 0,85^{a}$ & $+30,85 \pm 1,63^{a}$ & $+30,00 \pm 1,41^{\mathrm{a}}$ \\
\hline \multirow[t]{4}{*}{$C^{*}$} & T1 & $32,40 \pm 0,71^{\mathrm{a}}$ & $32,70 \pm 1,70^{\mathrm{a}}$ & $32,45 \pm 0,92^{\mathrm{a}}$ & $32,00 \pm 1,41^{\mathrm{a}}$ & $32,05 \pm 1,34^{\mathrm{a}}$ \\
\hline & T2 & $32,05 \pm 1,20^{\mathrm{a}}$ & $32,15 \pm 1,20^{\mathrm{a}}$ & $32,65 \pm 1,77^{\mathrm{a}}$ & $32,30 \pm 1,84^{\mathrm{a}}$ & $32,05 \pm 1,20^{\mathrm{a}}$ \\
\hline & T3 & $32,50 \pm 0,57^{\mathrm{a}}$ & $32,35 \pm 1,06^{\mathrm{a}}$ & $32,20 \pm 0,99^{\mathrm{a}}$ & $32,10 \pm 1,13^{\mathrm{a}}$ & $32,80 \pm 1,41^{\mathrm{a}}$ \\
\hline & T4 & $32,75 \pm 1,48^{\mathrm{a}}$ & $32,40 \pm 1,41^{\mathrm{a}}$ & $32,00 \pm 1,27^{\mathrm{a}}$ & $32,45 \pm 1,77^{\mathrm{a}}$ & $31,90 \pm 0,85^{\mathrm{a}}$ \\
\hline \multirow[t]{4}{*}{$\mathbf{H}$} & T1 & $73,10 \pm 1,27^{\mathrm{a}}$ & $72,75 \pm 0,92^{\mathrm{a}}$ & $73,15 \pm 1,63^{a}$ & $73,10 \pm 1,13^{\mathrm{a}}$ & $73,20 \pm 1,13^{\mathrm{a}}$ \\
\hline & T2 & $73,20 \pm 0,99^{\mathrm{a}}$ & $73,35 \pm 1,06^{a}$ & $72,85 \pm 0,21^{\mathrm{a}}$ & $73,20 \pm 0,85^{\mathrm{a}}$ & $73,50 \pm 0,99^{\mathrm{a}}$ \\
\hline & T3 & $73,40 \pm 0,71^{\mathrm{a}}$ & $73,15 \pm 0,92^{\mathrm{a}}$ & $73,15 \pm 0,92^{\mathrm{a}}$ & $73,40 \pm 1,13^{\mathrm{a}}$ & $73,55 \pm 1,20^{\mathrm{a}}$ \\
\hline & T4 & $73,05 \pm 0,92^{\mathrm{a}}$ & $73,15 \pm 1,20^{\mathrm{a}}$ & $72,70 \pm 0,99^{\mathrm{a}}$ & $73,05 \pm 1,06^{\mathrm{a}}$ & $73,05 \pm 1,20^{\mathrm{a}}$ \\
\hline
\end{tabular}

$\mathrm{n}=2$, resultados expressos em média \pm desvio-padrão. As médias seguidas pela mesma letra não diferem estatisticamente entre si. Foi aplicado o Teste de Tukey $(\mathrm{p} \leq 0,05)$. 


\subsection{Substâncias reativas ao ácido tiobarbitúrico (TBARS)}

O efeito da incorporação dos EPR, ESR e BHT sobre o valor de TBARS nos hambúrgueres de frango armazenados a $-18{ }^{\circ} \mathrm{C}$ e submetidos à cocção podem ser visualizados na Tabela 6. Os resultados foram expressos em $\mathrm{mg}$ de malonaldeído/Kg de amostra.

Tabela 6: Valores de TBARS expresso em mg de malonaldeído/Kg de amostra.

\begin{tabular}{lccccc}
\hline \multirow{2}{*}{$\begin{array}{l}\text { Tratamento } \\
\text { T1 }\end{array}$} & $\mathbf{0}$ & $\mathbf{7}$ & $\mathbf{1 4}$ & $\mathbf{2 1}$ & $\mathbf{2 8}$ \\
\cline { 2 - 6 } & $1,341 \pm 0,068^{\mathrm{a}}$ & $1,509 \pm 0,089^{\mathrm{a}}$ & $1,666 \pm 0,167^{\mathrm{a}}$ & $1,625 \pm 0,047^{\mathrm{a}}$ & $1,589 \pm 0,044^{\mathrm{a}}$ \\
\hline $\mathbf{T 2}$ & $0,674 \pm 0,139^{\mathrm{b}}$ & $0,787 \pm 0,170^{\mathrm{b}}$ & $0,585 \pm 0,136^{\mathrm{b}}$ & $0,707 \pm 0,174^{\mathrm{b}}$ & $0,640 \pm 0,030^{\mathrm{b}}$ \\
\hline $\mathbf{T 3}$ & $0,753 \pm 0,130^{\mathrm{b}}$ & $0,683 \pm 0,106^{\mathrm{b}}$ & $0,587 \pm 0,051^{\mathrm{b}}$ & $0,722 \pm 0,249^{\mathrm{b}}$ & $0,611 \pm 0,024^{\mathrm{b}}$ \\
\hline $\mathbf{T 4}$ & $0,616 \pm 0,064^{\mathrm{b}}$ & $0,623 \pm 0,068^{\mathrm{b}}$ & $0,719 \pm 0,007^{\mathrm{b}}$ & $0,693 \pm 0,010^{\mathrm{b}}$ & $0,703 \pm 0,086^{\mathrm{b}}$
\end{tabular}

$\mathrm{n}=2$, resultados expressos em média \pm desvio-padrão. As médias seguidas pela mesma letra não diferem estatisticamente entre si. Foi aplicado o Teste de Tukey $(\mathrm{p} \leq 0,05)$.

Foi observado que os valores de TBARS para os tratamentos T2, T3 e T4 não obtiveram diferença significativa $(p<0,05)$ no dia da análise, bem como ao longo dos 28 dias de armazenamento, ou seja, a variação de $\mathrm{mg}$ de molonaldeído/Kg de amostra no decorrer das análises não houve diferença significativa $(\mathrm{p}<0,05)$. Os valores oscilaram de 0,585 a 0,787 mg de molonaldeído/Kg de amostra. Já amostras do tratamento $\mathrm{T} 1$ apresentaram valores de malonaldeído superiores (1,341 a 1,666 mg de molonaldeído/Kg de amostra) em comparação com os demais tratamentos apresentando diferença significativa $(\mathrm{p} \leq 0,05)$.

Qin et al. (2013) [34] verificou em seu estudo sobre o potencial antioxidante de extratos da casca, suco e extrato da semente de romã em carne suína moída crua que os valores de TBARS aumentou em todas as amostras durante 12 dias armazenadas a $4{ }^{\circ} \mathrm{C}$; contudo, foi significativamente $(\mathrm{p}<0,05)$ inibida nos tratamentos com BHT $(0,40$ a 0,94$)$, extratos da casca $(0,41$ a 1,07$)$, suco $(0,48$ a 1,43$)$ e extrato da semente $(0,49$ a 1,49$)$ de romã em comparação com o grupo controle $(0,52$ a 1,98). Shahamirian et al. (2019) [4] em sua avaliação das características antibacterianas e antioxidantes do suco de romã e extrato da casca de romã em hambúrgueres bovino armazenados a $-18{ }^{\circ} \mathrm{C}$ durante 90 dias obteve ao final do estudo o valor de TBARS de aproximadamente 1,50 para a mostra controle e em torno de 1,05 para as amostras tratadas com BHT e suco de romã.

\section{CONCLUSÃO}

No presente estudo foi possível verificar um possível potencial antioxidante dos EPR e ESR e empregá-los, em substituição ao BHT, como promissoras alternativas para a estabilidade oxidativa de hambúrguer de frango. A adição dos extratos não provocou alteração da cor do produto cárneo em comparação com os demais tratamentos. Os extratos também contribuíram para que não houvesse decréscimo no valor do $\mathrm{pH}$ similar ao ocorrido no tratamento com o BHT. Apesar dos ESR e EPR apresentarem diferença significativa entre si $(p<0,05)$ na capacidade de sequestrar o radical $\mathrm{ABTS}^{+}$os mesmos, foram $100 \%$ efetivos no controle oxidativo verificado pelo método TBARS em comparação como BHT. Os EPR e ESR obtiveram similaridade ao BHT nas concentrações avaliadas, um ponto positivo. Contudo, há a necessidade de um estudo mais aprofundando acerca da dosagem ideal visto que no presente trabalho foi testado apenas um único valor. 


\section{REFERÊNCIAS BIBLIOGRÁFICAS}

1. Waraho T, Mcclements DJ, Decker EA. Review: Mechanisms of lipid oxidation in food dispersions. Trends Food Sci \& Technol. 2011 Jan;22(1):3-13, doi: 10.1016/j.tifs.2010.11.003.

2. Sampaio GR, Saldanha T, Soares RAM, Torres EAFS. Effect of natural antioxidant combinations on lipid oxidation in cooked chicken meat during refrigerated storage. Food Chem. 2012 Dec;135(3):13831390, doi: 10.1016/j.foodchem.2012.05.103.

3. Bhat ZF, Kumar S, Kumar L. Effect of Ocimum sanctum Linn (Tulsi) on the oxidative stability and storage quality of chicken sausages. Nutrit \& Food Sci. 2015 Jul;45(4):510-523, doi: 10.1108/NFS-012015-0002.

4. Shahamirian M, Eskandari MH, Niakousari M, Esteghlal S, Gahruie HH, Khaneghah AM. Incorporation of pomegranate rind powder extract and pomegranate juice into frozen burgers: oxidative stability, sensorial and microbiological characteristics. J Food Sci Technol. 2019 Mar;56(3):1174-1183, doi: 10.1007/s13197-019-03580-5.

5. Bordoni A, Francesca D. Chapter 11 - Poultry meat nutritive value and human health. In: Petracci M, Berri C, editors. Poultry quality evaluation: quality attributes and consumer values. 1st ed. United Kingdom: Woodhead Publishing, Elsevier; 2017. p. 279-290, doi: 10.1016/B978-0-08-100763-1.000118

6. Marcinkowska-Lesiak M, Zdanowska-Sąsiadek Ż, Stelmasiak A, Damaziak K, Michalczuk M, Poławska E, Wyrwisz J, Wierzbicka A. Effect of packaging method and cold-storage time on chicken meat quality. Cyta - J Food. 2016;14(1):41-46, doi: 10.1080/19476337.2015.1042054.

7. Özünlü O, Ergezer H, Gökçe R. Improving physicochemical, antioxidative and sensory quality of raw chicken meat by using acorn extracts. LWT - Food Sci Technol. 2018 Dec;98:477-484, doi: 10.1016/j.1wt.2018.09.007.

8. Kaderides K, Goula AM, Adamopoulos KG. A process for turning pomegranate peels into a valuable food ingredient using ultrasound-assisted extraction and encapsulation. Innov Food Sci \& Emerg Technol. 2015 Oct;31:204-215, doi: 10.1016/j.ifset.2015.08.006.

9. Cruz RG. Atividade antioxidante de extratos vegetais: estudo das condições de extração e aplicação em sistema lipídio [dissertação]. Piracicaba (SP): Escola Superior de Agricultura Luiz de Queiroz, Universidade de São Paulo; 2014.97 p.

10. Boroski M, Visentainer JV, Cottica SM, Morais DR. Antioxidantes: princípios e métodos analíticos. Curitiba: Appris; 2015. 141 p.

11. Singh B, Singh JP, Kaur A, Singh N. Phenolic compounds as beneficial phytochemicals in pomegranate (Punica granatum L.) peel: A review. Food Chem. 2018 Sep;261:75-86, doi: 10.1016/j.foodchem.2018.04.039.

12. Šavikin K, Živković J, Alimpić A, Zdunića G, Janković T, Duletić-Laušević S, Menković N. Activity guided fractionation of pomegranate extract and its antioxidant, antidiabetic and antineurodegenerative propertie. Ind Crops Products. 2018 Mar;113:142-149, doi: 10.1016/j.indcrop.2018.01.031.

13. Viuda-Martos M, Ruiz-Navajas Y, Fernández-López J, Sendra E, Sayas-Barberá E, Pérez-Álvarez JA. Antioxidant properties of pomegranate (Punica granatum L.) bagasses obtained as co-product in the juice extraction. Food Res Int. 2011 Jun;44(5):1217-1223, doi: 10.1016/j.foodres.2010.10.057.

14. Shaygannia E, Bahmani M, Zamanzad B, Rafieian-Kopaei M. A Review Study on Punica granatum L. J Evidence-Based Complementary \& Alternative Medicine. 2016;21(3):221-227, doi: $10.1177 / 2156587215598039$.

15. Naveena BM, Sen AR, Vaithiyanathan S, Babji Y, Kondaiah N. Comparative efficacy of pomegranate juice, pomegranate rind powder extract and BHT as antioxidants in cooked chicken patties. Meat Sci. 2008 jun;80(4):1304-1308, doi: 10.1016/j.meatsci.2008.06.00.

16. Devatkal SK, Narsaiah K, Borah A. The effect of salt, extract of kinnow and pomegranate fruit byproducts on colour and oxidative stability of raw chicken patties during refrigerated storage. J Food Sci Technol. 2011 Jul;48(4):472-477, doi: 10.1007/s13197-011-0256-9.

17. Turguta SS, Soyerb A, Işikçib F. Effect of pomegranate peel extract on lipid and protein oxidation in beef meatballs during refrigerated storage. Meat Sci. 2016 Jun;116:126-132, doi: 10.1016/j.meatsci.2016.02.011.

18. Firuzi MR, Niakousari M, Eskandari MH, Keramat M, Gahruie HH, Khaneghah AM. Incorporation of pomegranate juice concentrate and pomegranate rind powder extract to improve the oxidative stability of frankfurter during refrigerated storage. LWT - Food Sci Technol. 2019;102:237-245, doi: 10.1016/j.lwt.2018.12.048.

19. Singleton VL, Orthofer R, Lamuela- Raventós RM. Analysis of total phenols and other oxidation substrates and antioxidants by means of Folin-Ciocateau reagent. Methods Enzymol. 1999;299:152178. 
20. Brasil. Ministério da Agricultura, Pecuária e Abastecimento. Metodologia Científica: Determinação da atividade antioxidante total em frutas pela captura do radical livre ABTS. Comunicado técnico, n. 128, 2007.

21. Hautrive TP, Oliveira VR, Silva ARD, Terra NN, Campagnol PCB. Análise físico-química e sensorial de hambúrguer elaborado com carne de avestruz. Ciência e Tecnologia de Alimentos. 2008 Dez;28:95101, doi: 10.1590/S0101-20612008000500016.

22. Instituto Adolfo Lutz. Métodos físico-químicos para análise de alimentos. São Paulo: Instituto Adolfo Lutz; 2008. 1020 p. Versão eletrônica.

23. Konica Minolta Sensing Americas: Entendendo o Espaço de Cor L*a*b* [Internet]. Disponível em: http://sensing.konicaminolta.com.br/2013/11/entendendo-o-espaco-de-cor-lab/ [Acesso em 01 julho 2019].

24. Miyagusku L, Thomazini M, Kuaye AY, Castillo CJC. Avaliação do valor de tbars em coxas de frango irradiadas. Rev Inst Adolfo Lutz. 2007 Abr;66(1):45-49.

25. Ferreira AA. Peixe piranha (Pygocentres nattereri) do pantanal: composição em ácidos graxos e mudanças com o processamento e estocagem [dissertação]. Campo Grande (MS): Universidade Federal do Mato Grosso do Sul; 2010.

26. Damodaran S, Parkin KL, Fennema OR. Química de alimentos de Fennema. 4. ed. Porto Alegre: Artmed; 2010. 82 p.

27. Vital KL. Extração e Quantificação de Compostos Fenólicos na Romã [trabalho de conclusão de curso]. Assis (SP): Fundação Educacional do Município de Assis - FEMA; 2014. 44 p.

28. Nascimento KO, Batista E, Silva AA, Reis IP, Pires T, Barbosa MIMJ. Teor de compostos fenólicos totais em diferentes extratos de romã (Punica granatum 1.). Higiene Alimentar. 2013 Mar/Abr;27(218/219):1638-1641.

29. Silva JS, Bezerra RMN. Avaliar o potencial antioxidante "in vitro"do extrato de polpa, semente e casca da romã (Punica granatum). In: Anais do XX Congresso interno de iniciação científica da Unicamp; 24-26 Out 2012; Campinas, São Paulo, Brasil. Campinas, SP: UNICAMP/Pró-Reitoria de Pesquisa; 2012.

30. Pierotto MF, Nott BL, Weimer P, Rossi R, Castilhos J. Compostos fenólicos totais e atividade antioxidante de diferentes extratos de romã (Punica granatum L.). In: Anais do Simpósio Latino Americano de Ciência dos Alimentos; 04-07 Nov 2017; Campinas, São Paulo, Brasil. São Paulo: FEA/UNICAMP; 2017.

31. Borba CM, Oliveira VR, Montenegro KR, Hertz PF, Venzke JG. Avaliação físico-quimica de hambúrguer de carne bovina e de frango submetidos a diferentes tratamentos térmicos. Alim Nutr. 2013 Jan;24(1):21-27.

32. Torquilho DCC, Feijó MBS, Fernandes ML, Fonseca ABM. Influência do modo de preparo de produtos cárneos industrializados na composição centesimal e valor energético. In: Anais do XI Congresso Brasileiro de Higienistas de Alimentos. 2011; Salvador, Bahia, Brasil. São Paulo: Higiene Alimentar; 2011.

33. Brasil. Ministério da Saúde. Agência Nacional de Vigilância Sanitária. Regulamento Técnico de Identidade e Qualidade do Hambúrguer. Instrução Normativa no 20/2000.

34. Qin Y, Zhang Z, Li L, Xiong W, Shi J, Zhao T, Fan J. Antioxidant Effect of Pomegranate Rind Powder Extract, Pomegranate Juice, and Pomegranate Seed Powder Extract as Antioxidants in Raw Ground Pork Meat. Food Sci Biotechnol. 2013 Aug;22(4):1063-1069, doi: 10.1007/s10068-013-0184-8. 